\title{
Unrealistic Optimism Among a Sample of University Students in Egypt and Saudi Arabia (Cross-cultural Study)
}

\author{
Mokhtar Ahmad Al-Kayal, Ph.D. ${ }^{1} \quad$ Mohammad Ismail Sayed Hemeda, Ph.D ${ }^{1}$ \\ Mohammad Ali Haiba, Ph.D. ${ }^{1}$ Walid Hassan Ashour El Khateeb, Ph.D. ${ }^{2}$ \\ 1. Educational Psychology Department, Faculty of Education, Ain Sham University \\ 2. Educational Psychology Department, Faculty of Education, Ain Sham University and Jouf University
}

\begin{abstract}
The present study considered as cross-cultural study attempts to examine the psychometric properties of unrealistic optimism in light of the scale used in the study for Egyptian and Saudi Arabia university students and Is the measurement equivalent of the scale terms for Egyptian and Saudi Arabia university students? . A total of (336) male students from university in Egypt and in Saudi Arabia from different specialties completed the scale of unrealistic optimism (prepared by the researchers), Confirmatory factor analysis (CFA) is used. The results indicate that the structure of model of unrealistic optimism is fit and the scale has appropriate psychometric properties. And the result indicate that the unrealistic optimism scale have the same structure in Egypt and Saudi Arabia , that means the factorial invariance of unrealistic optimism in Egypt and Saudi Arabia .
\end{abstract}

Keywords: Unrealistic optimism, Reliability, Construct validity, Cultural differences.

DOI: $10.7176 /$ RHSS/9-24-12

Publication date: December $31^{\text {st }} 2019$

\section{Introduction}

The concept of optimism is one of the new concepts that is frequently used among researchers interested in the field of positive psychology (Jaber, El Aasar, \& Youssef, 2013, 278).

Optimism is generally an energizing and important factor in the life of the individual, linked to the positive outlook of the future, while pessimism is associated with the negative outlook on life, and leads to sadness and despair and fear of decline, and makes one believes that many things are a source of threat to him, which drives him to move away from many situations. Then he does not solve his problems, or overcome obstacles, and then is incompatible with his environment (Al-Qudah, Hilat, \& Al-Zaboun, 2013, 1542).

The concepts of optimism and pessimism have been defined in a number of different ways. Optimism and pessimism defined as generalized positive and negative outcome expectancies, they also defined as a positive and negative outlook on life. Both of these definitions imply that optimism and pessimism are relatively stable traits that are consistent across time and situations (Ji, Zhang, Usborne, \& Guan, 2004, 26).

Despite the importance of the concept of optimism in human life in general, the concept of optimism and pessimism and the factors most closely associated with them did not receive sufficient attention in psychological studies, as optimism and positive thinking are essential to the public health of the individual, and pessimism , negative view of events and the Negative moods are closely related to some of the causes of poor social harmony and mental disorders that individuals, youth and adolescents, may experience. Optimists have a sense of self-worth and efficiency, evaluate their performance more positively, while pessimists assess their performance more negatively and underestimate (El-Sherbini, \& Damanhouri, 2010, 1106). In the following, we will display for the main terms of the study:

\subsection{Optimism}

Mashashu $(2011,27)$ pointed out that an optimistic person can deal with negative information, events and situations more positively and more successfully than pessimists. Optimistic person able to deal with information and results, has coping skills, able to solve problems, with high self-confidence, and able to achieve his goals.

Optimism is the expectation of success in both the near future and the distant future (Seligman, 2010, 8).

Jaber et al., $(2013,277)$ define optimism as the positive expectation of the individual towards himself and others, as well as the perception of things around him in a positive way and expect the best in present and in future.

Suleiman $(2016,552)$ defines optimism as the tendency to expect positive results in life.

The current researchers define optimism as the tendency to expect the positive expectation of the individual towards himself and others in present and in future.

\subsection{Unrealistic optimism}

The concept of unrealistic optimism can be related to theory and research on "positive - negative asymmetry" in cognition and affect reviewed by Peeters and Czapinski (1990) and further developed by them into a theory of subjective well-being. According to the theory, optimism is part and parcel of a biopsychological strategy to survive in an environment with a larger potential for negative than for positive life outcomes (Peeters, Cammaert, 
\& Czapinski, ,1997,24).

Ever since its original demonstration by Weinstein (1980) a great deal of empirical work and theoretical attention has been devoted to the phenomenon of unrealistic optimism. The term refers to a bias whereby "people rate negative events as less likely to happen to themselves than to the average person and positive events as more likely to happen to themselves than to the average person ( Joshi \& Carter,2013,1).

It has been proposed that individuals tend to be unrealistically optimistic about their likelihood of experiencing positive life events and of not experiencing negative life events in comparison to their peers. Convincing people to adopt prudent health behaviors may be more difficult if they believe they are either not susceptible to a disease or less susceptible than others. Individuals in general believe that they are less likely than other people to experience negative health outcomes. Researchers found that there is a tendency for individuals to indicate that other people have a higher risk than themselves for experiencing a negative life event. This tendency shows an optimistic bias. Identifying such optimistic biases in personal risk perceptions is necessary because they may seriously hinder efforts to promote risk-reducing behaviors (Reyes-Velázquez \& Sealey-Potts,2015,6)

Researchers in the field of personality have been interested in the concept of unrealistic optimism in recent years, they differentiate between optimism and unrealistic optimism, as optimism is a comprehensive cognitive approach and tendency within the individual to the general expectation of the occurrence of positive things rather than the occurrence of negative things and to have a positive perception of the self and the outside world, While unrealistic optimism is defined as a tendency that makes an individual expect more positive things from happening in reality and benefit from them, unrealistic optimism viewed as defensive mechanism that Ego used to reduce anxiety caused by fear of the dangers (Boutbal, 2012.108).

Al-Ansari $(2001,201)$ found that researchers view unrealistic optimism as one of the defensive mechanisms that ego uses to reduce anxiety, while others interpret it as an inherently selfish tendency that affects their beliefs about anxiety and danger, they believe that these events may happen to others without them, and others believe that unrealistic optimism is the result of cognitive error in which the individual falls. Others consider it to be a result of low intelligence or a lack of certain mental skills leading the individual to underestimate the potential risks or events that are likely to be exposed.

People are considered unrealistically optimistic if they predict that a personal future outcome will be more favorable than that suggested by a relevant, objective standard. Unrealistic optimism also occurs when people unduly predict that their personal outcomes will be more favorable than the outcomes of peers. The concept is distinct both empirically and conceptually from dispositional optimism, a personality trait representing generally positive expectations about the future (Shepperd, Waters, Weinstein, \& Klein,2015, 232-233).

Unrealistic optimism has been called as comparative optimism or defensive optimism (Sereen, 2014,169). It defined by (Al-Ansari, 2001, 194) as an individual's belief in his ability to be optimistic towards events without logical justifications or facts that lead to this belief.

Unrealistic optimism is not a personal trait, but a systematic cognitive distortion to take into account the probability of coping with negative events. It is the result of joint efforts of two mechanisms, the first of which relates to cognitive factors such as lack of information and poor individual cognitive skills. The second is of a catalytic nature, closely linked to the defense of self-esteem (Conversano, Rotondo, Lensi, Vista, Arpone, \& Reda, 2010,25).

Unrealistic optimism is one's belief that positive things can happen more than they actually are, and his belief that negative things can't happen less than they actually are (Mashashu, 2011, 27), (Al-Enezi, 2015, 207).

It is defined as a bias in which people believe that their negative events as less likely to occur than the average person and positive events that are more likely to occur to themselves than the average person (Harris \& Hahn, 2011,135).

Unrealistic optimism is an internal tendency that often leads the individual to expect more positive things to happen and less negative ones to happen (Al-Qudah et al., 2013, 1547).

Many researchers see unrealistic optimism as an example of self-reinforcing bias in general and optimistic bias even if it is unrealistic. Conversely, pessimistic bias is thought to cause depression (Joshi \& Carter, 2013, 1).

Al-Qudah et al. $(2013,1549)$ define unrealistic optimism as an internal tendency that often leads an individual to expect more positive things to happen than to happen and less negative things to happen.

Sereen $(2014,166)$ defines unrealistic optimism as an individual's belief that bad events may happen more on others than on himself, and that good events happen more to him than to others.

Unrealistic optimism is a tendency to form biased beliefs, often wrong, but they have great benefits, because they increase psychological well-being, contribute to mental and physical health support, and support productivity and motivation (Bortolotti \& Antrobus, 2015, 195).

Jefferson, Bortolotti, \& Kuzmanovic $(2016,2)$ define it as permanent patterns of beliefs about the self, the world, and the future that make things look better than they are, so they can be understood as systematic tendencies to either adopt and maintain optimistic beliefs about self or to make optimistic predictions about self where we can understand predictions as beliefs about what will happen or what is likely to happen. 
It can be said that unrealistic optimism occurs when the individual realizes that his personal results are more positive than the results of others in similar circumstances, and that their relative risk for him is more positive than the risk of others. This depends on the ability to control the event and individual's tendency to focus emotionally on the result.

The current researchers define unrealistic optimism as an individual's belief that bad events may happen more on others than on himself, and that good events happen more to him than to others.

\subsection{Types of unrealistic optimism}

Researchers distinguish between two types of unrealistic optimism that differ in the standard against which personal predictions are compared. The first type is unrealistic absolute optimism, which refers to an unjustified belief that a personal outcome will be more favorable than the outcomes indicated by a quantitative objective standard (such as epidemiological or base-rate data). Researchers have demonstrated unrealistic absolute optimism using many criteria. For example, several studies compared personal predictions with the outcomes that actually transpired, such as exam grades or starting salaries after college graduation. Other studies demonstrate that financial advisors are overly optimistic in their economic predictions and that people in general are quite unrealistic in their estimates of the time it will take to complete a task, a misjudgment known as the planning fallacy. Investigations have also demonstrated unrealistic absolute optimism when comparing people's estimates with population base rates. Researchers have shown similar patterns of unrealistic optimism in people's estimates of the likelihood that they will experience a divorce, sexually transmitted disease, or unplanned pregnancy.

The second type is unrealistic comparative optimism, which refers to the erroneous estimate that one's personal outcomes will be more favorable than the outcomes of peers. Researchers use two general approaches to document unrealistic comparative optimism. The first is when an individual incorrectly judges that his or her risk is less than that of other people. For example, study participants estimated whether they were more likely, less likely, or equally likely to have a fatal heart attack than the average person. The researchers then compared these estimates with the predictions of an pidemiologically-based personalized heart attack risk assessment algorithm to determine whether participants were indeed more, less, or equally likely to have a fatal heart attack compared with the average person. The comparison revealed that $56 \%$ of participants were unrealistically optimistic, $25 \%$ were unrealistically pessimistic, and 19\% were accurate. Other studies have revealed similar findings for breast cancer and smoking related diseases future )Shepperd, Waters, Weinstein, \& Klein,2015, 233)

Comparative optimism refers to the belief that positive events are more likely and negative events are less likely in one's future than in others' future. Absolute optimism refers to the belief that positive events are likely and negative events are unlikely in one's future. Comparative optimism occurs in conjunction with a variety of life events, including events that people spontaneously think of while imagining their future and events that are presented to them, among both women and men, in different ages, and across cultures (Hoorens, Damme, HelwegLarsen, \& Sedikides,2017,46).

\subsection{Assessment of unrealistic optimism}

Unrealistic optimism is typically assessed using either a direct or indirect elicitation method. In the direct approach respondents provide a single comparative rating (e.g. 'compared with a person the same age and gender as you, how likely are you to experience [a negative health outcome]'). The indirect approach requires the respondent to make two judgements. One judgement relates to the typical other's risk level (e.g. 'How likely is the average person to experience ...') and this is compared with a rating for own risk level (e.g. 'How likely are you to experience ...'). The difference between these two risk ratings is the comparative risk level ( Hevey, French, Marteau, \& Sutton, 2009, 373).

The different measures may yield different levels of unrealistic optimism, raising concerns over the extent to which reported levels of unrealistic optimism may be artefacts of the methods employed. Otten \& Van der Pligt (1996) reported that the direct method was associated with higher levels of optimism for negative life events than the indirect method in a sample of students. In contrast, Sutton (2002) found that the direct method revealed a pessimistic bias among adult smokers rating their risk of lung cancer, whereas the indirect method revealed an optimistic bias.

\subsection{Unrealistic optimism and cultural differences}

Recent research has suggested that the cultural environments in which people live affect the way they perceive the world. Optimism and pessimism have been investigated across cultures. With regards to dispositional optimism, North Americans have been found to be more optimistic than Asians. However, when dispositional optimism and pessimism were measured as separate and independent constructs on the Extended Life Orientation Test, Asian Americans were found to be more pessimistic, but not less optimistic, than European Americans (Ji, Zhang, Usborne, \& Guan, 2004,26- 27).

Most studies investigating comparative optimism across cultures using the direct method have generally 
found evidence of unrealistic optimism in both Easterners and Westerners) Rose, Endo, Windschitl, \& Suls, 2008, 1238).

With regards to unrealistic optimism, Heine \& Lehmak (1995) conducted a study aimed to investigate cultural differences and differences in unrealistic optimism on a sample of university students consisting of (196) Japanese students and (314) Canadian students. They found that Canadians showed significantly more unrealistic optimism than did Japanese participants.

Rose, Endo, Windschitl, \& Suls $(2008,1237-1238)$ stated that in some studies, people from Western cultures (i.e., North Americans) tended to be more unrealistically optimistic than people from Eastern cultures (i.e., Japan) when comparing their chances of experiencing negative events to the average person's. In cross-cultural studies using the indirect method to measure comparative optimism (which happen to be very few), Westerners tend to be unrealistically optimistic, whereas Easterners tend to be unrealistically pessimistic or neutral (neither optimistic nor pessimistic).

Ji, Zhang, Usborne, \& Guan (2004) predicted ,based on early research, that the Chinese may be more optimistic and less pessimistic than North Americans in response to negative life events. A survey was conducted to investigate optimism cross culturally in the context of the severe acute respiratory syndrome (SARS) outbreaks in Canada and China. One hundred and four Chinese students from Beijing and (35) European Canadians from Toronto participated in the survey. Chinese participants were volunteers recruited on the campus of Beijing University. Chinese students in Beijing and European Canadians in Toronto answered questions about their perceptions of SARS. No significant cultural difference was found on dispositional optimism, as measured by the Revised Life Orientation Test (LOT-R). Unrealistic optimism was measured in the context of SARS. Both groups demonstrated unrealistic optimism (i.e. reporting that the self was less likely than an average person to get infected with SARS). Such optimistic bias was stronger among Chinese than among Canadians. Compared to the actual infection rates in Beijing and Toronto, both Chinese and Canadian participants overestimated their own chances of getting infected, indicating that they were being pessimistic. Indeed, Chinese were less pessimistic than Canadians. In addition, even though the Chinese reported more inconvenience brought by SARS than did Canadians, they also reported more positive changes brought by SARS, reflecting the Chinese dialectical views of events.

Peeters, Cammaert, \& Czapinski (1997) conducted a study aimed to identify cultural differences in unrealistic optimism on a sample of Belgians, Moroccans and Poles. It consisted of (670) Poles, (337) Belgians, and (125) Moroccans aged 17 - 37-year-old. Weinstein scale of unrealistic optimism applied. The results of the study revealed cultural differences in unrealistic optimism, with Poles having the highest rates, followed by Belgians and Moroccans.

(Plan,2015)provides a theoretical overview, including a neurobiological perspective of well-being, eudaimonic well-being, optimism, pessimism and cultural differences between Western and Eastern societies. In addition, an empirical study investigated these concepts in Japanese and Swedish participants. Definitional problems and scarce neurobiological findings are two current problems to date within research on WB, EWB and cultural differences especially when looking at comparisons between Europe and East Asia. Interpretations and conclusions are therefore hard and tentative to make as more research is yet needed. This thesis empirical part therefore investigated the association between these concepts. In the best of the authors knowledge have this type of explorative study never been done before. 142 Swedish participants and 68 Japanese participants between the ages of 20 to 40 answered the self-reporting questionnaires; revised life orientation test (LOT-R), psychological well-being scale (PWBS) and the minimalist well-being scale (MWBS). The findings demonstrate that Swedish people report higher levels of optimism compared with Japanese people whom in turn report higher levels of pessimism when measured with LOT-R. Findings further demonstrate that Swedish people report higher levels of (EWB) when measured with (PWBS). In comparison do Japanese people report higher levels of (EWB) when measured with MWBS. A difference was found in response pattern between MWBS and (PWBS).

Rose, Endo, Windschitl, \& Suls (2008) conducted a study tested whether the method used to assess unrealistic optimism influenced cross-cultural patterns in the United States and Japan. Participants for the main study were (127) students from the University of Iowa (United States) and (123) students from Kansai University (Japan). Students from both countries participated in the study as part of a psychology course requirement and were comparable in age (U.S. sample $\mathrm{M}=18.63$ years, $\mathrm{SD}=1.40$; Japanese sample $\mathrm{M}=20.44$ years, $\mathrm{SD}=3.99$ ). The results showed that the direct method (a single comparison judgment between self and peers) produced similar patterns across cultures because of cognitive biases (e.g., egocentrism); specifically, participants were unrealistically optimistic about experiencing infrequent/negative events but pessimistic about experiencing frequent/ negative events. However, the indirect method (separate self- and peer judgments) produced different patterns across cultures because culturally specific motivational biases emerged using this method; specifically, the U.S. sample was more unrealistically optimistic than the Japanese sample.

Joshi \& Carter (2013) in their study extends enquiry to a different non-Western culture. Two hundred and eighty seven middle aged and middle income participants (200 in India, 87 in England) rated 11 positive and 11 
negative events in terms of the chances of each event occurring in "their own life," and the chances of each event occurring in the lives of "people like them." Comparative optimism was shown for bad events, with Indian participants showing higher levels of optimism than English participants. The position regarding comparative optimism for good events was more complex. In India those of higher socioeconomic status (SES) were optimistic, while those of lower SES were on average pessimistic. Overall, English participants showed neither optimism nor pessimism for good events. The results, whose clinical relevance is discussed, suggest that the expression of unrealistic optimism is shaped by interplay of culture and socioeconomic circumstance.

It can be seen from the results of the prior studies that the cultural dimension may play a core role in making differences in unrealistic optimism, and this is what the present study is trying to examine.

The present study was undertaken as a cross-cultural examination of unrealistic optimism in two samples, university students in Egypt and university students in Saudi Arabia, as well as to provide farther evidence supporting the construct validity of unrealistic optimism. Our attempt to compare Saudi Arabia and Egyptian university students' reports on unrealistic optimism is based on the different features that characterize each of these cultures.

The research questions addressed were:

1- What are the psychometric properties of unrealistic optimism in light of the scale used in the study for Egyptian and Saudi Arabia university students?

2- Is the measurement equivalent of the scale terms for Egyptian and Saudi Arabia university students?

\section{Methodology}

To answer the study questions, it was necessary to prepare an appropriate scale for unrealistic optimism, then apply it on both samples. Finally, the data was collected and analyzed.

\section{Participants}

The population of the study consisted of :

Egyptian sample: A total of (156) male university students from the first, the second, the third and the fourth year from different specialties included: Arabic, English, chemistry, physics, mathematics, psychology, history, geography and philosophy at faculty of education, Ain sham university, completed scale of unrealistic optimism, ranged in age from 18 to 21 with an average age (19.2) and S.D.(1.7).

Saudi Arabia sample : A total of (180) male university students from first to eight level from different specialties included: Arabic, English, chemistry, physics, mathematics, Islamic studies and business administration at faculty of sciences and arts in Qurayyat, Jouf University, completed scale of unrealistic optimism, ranged in age from 18 to 22 with an average age ( 19.9 ) and S. D. ( 1.4 ).

\section{Instrument}

The researchers prepared unrealistic optimism scale that used in the current study, to achieve that they used some prior scales of unrealistic optimism such as unrealistic optimism scales prepared by Weinstein (1980), AlAnsari (2001), Sereen (2011), Mashashu (2011), The scale consists of (22) items, including (11) items for pleasant or positive events and (11) items for unpleasant or negative events. Items numbers for pleasant or positive events are $(1,3,5,7,9,11,13,15,17,19,21)$, where items numbers for unpleasant or negative events are $(2,4,6,8,10$, $12,14,16,18,20,22)$. It is answered using a graded 8-point Likert scale.

\section{Results}

\section{Confirmatory factor analysis of unrealistic optimism:}

Confirmatory factor analysis (CFA) is used to study the relationships between observed values and latent constructs and comprises the measurement model portion of a structural equation model. CFA additionally, examine the stability of the theorized factor structure, to provide information for measure refinement and to establish the uni- dimensionality of the construct.

Confirmatory factor analysis was conducted in order to further evaluate the items and their structure. The confirmatory factor analysis was done using Analysis of AMOS) software to unrealistic optimism. The measurement model for constructs was examined. For approximate fit indexes, Goodness of Fit Index (GFI), Adjusted Goodness of Fit Index (AGFI), Normed fit index (NFI), Relative Fit Index (RFI), Incremental Fit Index (IFI), Tucker-Lewis fit index (TFI) and Comparative Fit Index (CFI) of above 0.9 would indicate model fit. More specifically, for Chi-square/df, a value close to 1 is ideal where however values below 5 are considered acceptable. For RMSEA (Root Mean Square Error Approximation), its value should be less than 0.05 although less than 0.1 is suggested to indicate good fit, for NFI, RFI, IFI, TLI and CFI, their values range along a $0-1$ continuum, where values above 0.9 typically correspond to good fit .

It is recommended to use multiple and different fit indices where it is suggested to use at least three indexes to assess the model fit. It is suggested to involve at least one absolute fit index and one incremental fit index in 
addition to ratio of chi-square to degrees of freedom (normed chi-square) to define the fit of the model. In this research, RMSEA was used as the absolute fit index as it is one of the most informative criteria as to an absolute fit, CFI (Comparative Fit Index) was used as the incremental fit index as it has the ability to adjust for model complexity, in addition to NFI and RFI.

Confirmatory Factor Analysis was used to confirm one factor model of unrealistic optimism in the total sample of Egypt and Saudi Arabia.

Table (1): Model Fit of unrealistic optimism in the total sample of Egypt and Saudi Arabia

\begin{tabular}{|c|c|c|}
\hline & Stats & One factor model \\
\hline & Chi-square & 351.7 \\
\hline & Probability level & 0.000 \\
\hline \multirow{4}{*}{$\begin{array}{c}\text { Model Fit } \\
\text { Summary } \\
\text { (CMIN) }\end{array}$} & NPAR & 119 \\
\hline & CMIN & 351.7 \\
\hline & DF & 134 \\
\hline & CMIN/DF & 2.62 \\
\hline \multirow{5}{*}{$\begin{array}{c}\text { Baseline } \\
\text { Comparisons }\end{array}$} & NFI & 0.92 \\
\hline & RFI & 0.86 \\
\hline & IFI & 0.9 \\
\hline & TLI & 0.91 \\
\hline & CFI & 0.95 \\
\hline \multirow{2}{*}{ RMSEA } & RMSEA & .07 \\
\hline & PCLOSE & 0.000 \\
\hline
\end{tabular}

First, the measurement model showed a good fit to the data with CMIN/DF of 2.62, CFI of 0.95, NFI of 0.92, RFI of 0.86 , IFI of 0.90 , TLI of 0.91 and RMSEA of 0.07. Second, all factor loadings of the measurement model were examined. They all were above 0.4 and statistically significant $(\mathrm{p}<0.05)$, modification indices were examined in order to improve the model fit. Modification indices suggested creating a covariance between errors on unrealistic optimism, where the measurement model resulted in a better fit.

Table(2): Regression, Std. regression of unrealistic optimism

\begin{tabular}{|c|c|c|c|c|c|c|c|}
\hline & & & Std. Estimate & Estimate & S.E. & C.R. & $\mathrm{P}$ \\
\hline A10 & $<---$ & Unrealistic optimism & 0.493 & 1.008 & .142 & 7.124 & $* * *$ \\
\hline A9 & $<---$ & Unrealistic optimism & 0.52 & .834 & .112 & 7.430 & $* * *$ \\
\hline A8 & $<---$ & Unrealistic optimism & 0.52 & 1.099 & .149 & 7.370 & $* * *$ \\
\hline A7 & $<---$ & Unrealistic optimism & 0.481 & .768 & .110 & 6.964 & $* * *$ \\
\hline A6 & $<---$ & Unrealistic optimism & 0.493 & 1.075 & .154 & 6.973 & $* * *$ \\
\hline A5 & $<---$ & Unrealistic optimism & 0.655 & .896 & .105 & 8.508 & $* * *$ \\
\hline A4 & $<---$ & Unrealistic optimism & 0.478 & .915 & .131 & 6.955 & $* * *$ \\
\hline A3 & $<---$ & Unrealistic optimism & 0.642 & 1.171 & .140 & 8.341 & $* * *$ \\
\hline A2 & $<---$ & Unrealistic optimism & 0.568 & 1.201 & .143 & 8.397 & $* * *$ \\
\hline A1 & $<---$ & Unrealistic optimism & 0.507 & 1.000 & - & - & - \\
\hline a11 & $<---$ & Unrealistic optimism & 0.571 & .795 & .100 & 7.911 & $* * *$ \\
\hline a12 & $<---$ & Unrealistic optimism & 0.636 & 1.335 & .124 & 10.798 & $* * *$ \\
\hline a13 & $<---$ & Unrealistic optimism & 0.698 & 1.244 & .131 & 9.484 & $* * *$ \\
\hline a14 & $<---$ & Unrealistic optimism & 0.627 & 1.294 & .156 & 8.285 & $* * *$ \\
\hline a15 & $<---$ & Unrealistic optimism & 0.652 & .939 & .110 & 8.523 & $* * *$ \\
\hline a16 & $<---$ & Unrealistic optimism & 0.536 & 1.000 & .134 & 7.442 & $* * *$ \\
\hline a17 & $<---$ & Unrealistic optimism & 0.501 & .856 & .120 & 7.146 & $* * *$ \\
\hline a18 & $<---$ & Unrealistic optimism & 0.316 & .567 & .114 & 4.984 & $* * *$ \\
\hline a19 & $<---$ & Unrealistic optimism & 0.682 & 1.074 & .113 & 9.471 & $* * *$ \\
\hline a20 & $<---$ & Unrealistic optimism & 0.559 & .802 & .103 & 7.795 & $* * *$ \\
\hline a21 & $<---$ & Unrealistic optimism & 0.521 & .986 & .135 & 7.314 & $* * *$ \\
\hline a22 & $<---$ & Unrealistic optimism & 0.599 & .814 & .100 & 8.112 & $* * *$ \\
\hline
\end{tabular}

The CFA model should be purified through dropping items with low factor loadings, loadings above .6 to be "high" and loadings below .4 "low". All of the path coefficients were statistically significant, and all items loaded well above 0.4 , the item " 18 "has a factor loading of 0.316 , below the cutoff of 0.4 . Therefore it was dropped from the scale where standardized regression weight (standardized loading factor) estimates signify that the 
indicator variables are significant and representative of their latent variable.

\section{Construct Reliability:}

After conducting confirmatory factor analysis, reliability of items was estimated by calculating Cronbach's alpha value. The Cronbach's alpha value for scale was 0.86 and considered suitable.

\section{Comparison the factorial structure of unrealistic optimism in Egypt and Saudi Arabia:}

Confirmatory analysis was used to investigate factorial invariance unrealistic optimism in Egypt and Saudi Arabia, two unrealistic optimism models were examined, one with a free factor loading estimated in Egypt and Saudi Arabia (model1) and the other with the equality of factor loading in Egypt and Saudi Arabia (model2).

Table (3): Model comparison of unrealistic optimism in Egypt and Saudi Arabia

\begin{tabular}{|c|c|c|c|c|}
\hline & Stats & Free factor loading(model1) & Equality of factor loading(model2) & Diff. \\
\hline \multirow{4}{*}{$\begin{array}{c}\text { Model Fit } \\
\text { Summary } \\
\text { (CMIN) }\end{array}$} & Chi-square & 1168.52 & 1197.31 & 28.79 \\
\cline { 2 - 5 } & Probability level & 0.000 & 0.000 & - \\
\cline { 2 - 5 } & NPAR & 216 & 196 & 20 \\
\cline { 2 - 5 } & CMIN & 1168.52 & 1197.31 & 28.79 \\
\hline \multirow{3}{*}{$\begin{array}{c}\text { Baseline } \\
\text { Comparisons }\end{array}$} & DF & 246 & 266 & 20 \\
\cline { 2 - 6 } & RFI & 4.75 & 4.50 & 0.25 \\
\cline { 2 - 6 } & IFI & 0.79 & 0.60 & 0.01 \\
\cline { 2 - 6 } & TLI & 0.66 & 0.86 & 0.02 \\
\hline \multirow{2}{*}{ RMSEA } & RMSEA & 0.84 & 0.89 & 0.01 \\
\cline { 2 - 6 } & PCLOSE & 0.88 & 0.81 & 0.02 \\
\hline
\end{tabular}

A chi-square difference test, with $\chi^{2}$ diff $=28.79$ and $\mathrm{df}_{\text {diff }}=20,(\chi 2$ table $=31.41$ at 0.05 level and 37.56 at 0.01 level) reveals that no significant difference between model1 and model 2 , that means the factorial invariance of unrealistic optimism in Egypt and Saudi Arabia is the same.

\section{Conclusion}

This result can be explained that the items loading of unrealistic optimism do not differ in the Egyptian environment from the Saudi environment among university students. It is explained in the light of many reasons and facts that the Egyptian and Saudi cultures are close and the most university students in Egypt and Saudi Arabia have the same unrealistic optimism which may be related to the current economic conditions in Egypt and Saudi Arabia, The great similarity in culture between the two societies, especially as they are Arab societies, The work of a large number of Egyptians in Saudi Arabia and the nature of the Arab culture and religious culture that call for optimism.

\section{References}

Al-Ansari, B. (2001). Preparation of unrealistic optimism scale for a sample of male and female students in Kuwait, Psychological Studies, 11 (2), 194-243.( In Arabic)

Al-Enezi, A. (2015). The role of unrealistic optimism in the relationship between health beliefs and smoking behavior for university youth, International Journal of Specialized Education, Semat House for Studies and Research, 9 (4), 203-220. ( In Arabic)

Al-Qudah, M., Hilat, M., \& Al-Zaboun, M. (2013). Unrealistic optimism among students for educational sciences faculties in Jordanian universities and its relationship with some social variables, Studies in Educational Sciences, 40 (2), 1542-1554. ( In Arabic)

Bortolotti, L., \& Antrobus, M. (2015). Costs and benefits of realism and optimism. Current Opinion in Psychiatry, 28(2), 194-198.

Boutbal, S. (2012). The role of unrealistic optimism in committing traffic accidents among young drivers. Journal of Psychological and Educational Studies, 9, 101-124. ( In Arabic)

Conversano, C., Rotondo, A., Lensi, E., Vista, V., Arpone, F., \& Reda, M, (2010). Optimism and its impact on mental and physical well-being. Clinical Practice \& Epidemiology in Mental Health, 6, 25-29.

El Fangary, H. (2007). The relationship between unrealistic optimism, health problems and anxiety. Childhood Studies, 37 (10), 25-38. ( In Arabic)

El-Sherbini, Z., \& Damanhouri, R. (2010). Optimism as a reflection of parents' interaction with their adolescent children. Journal of the Faculty of Arts, 23 (3), 1101-1143. ( In Arabic)

Harris, L., \& Hahn, U. (2011). Unrealistic optimism about future life events : A cautionary note. Psychological 
Review, 118(1), 135-154

Heine, J., \& Lehmak, R. (1995). Cultural variation in unrealistic optimism : Does the West feel more invulnerable than the East?. Journal of Personality and Social Psychology, 68( 4 ), 595- 607.

Hevey, D., French, D., Marteau, T., \& Sutton, S. (2009). Assessing unrealistic optimism : Impact of different approaches to measuring susceptibility to diabetes. Journal of Health Psychology, 14(3), 372-377.

Hoorens, V., Damme, C.,Helweg-Larsen, M., \& Sedikides, C. (2017). The hubris hypothesis : The downside of comparative optimism displays. Consciousness and Cognition, 50, 45-55.

Jaber, Z., El Aasar, S., \& Youssef, M. (2013). Optimism and pessimism and their relationship with the locus of control for university students (from both gender). Journal of Scientific Research in Arts, 14 (4), 271-305. ( In Arabic)

Jefferson, A., Bortolotti, L., \& Kuzmanovic, B. (2016).What is unrealistic optimism?. Consciousness and Cognition, $\underline{\text { http: } / / \text { dx.doi.org/10.1016/j.concog.2016.10.005 }}$

Ji, L., Zhang, Z., Usborne, E., \& Guan, Y. (2004) . Optimism across cultures : In response to the severe acute respiratory syndrome outbreak. Asian Journal of Social Psychology, 7, 25-34.

Joshi, M. \& Carter, W. (2013). Unrealistic optimism : East and West?. Original Research Article, 4 (6),1-16.

Mashashu, K. (2011). The relationship of unrealistic optimism with smoking behavior for smokers. Unpublished Master Thesis, Faculty of Human and Social Sciences, University of Haj Lakhdar, Algeria. ( In Arabic)

Otten, W., \& Van der Pligt, J. (1996). Context effects in the measurement of unrealistic optimism in probability judgments. Journal of Social and Clinical Psychology, 15, 80-101.

Peeters, G., Cammaert, M., \& Czapinski, J. (1997). Unrealistic optimism and positive - negative asymmetry : A conceptual and cross - cultural study of interrelationships between optimism and realism. International Journal of Psychology, 32( 1 ), 23- 34.

Plan , A. (2015). Cultural differences in optimism, pessimism and eudaimonic well-being from a neurobiological perspective. Submitted to the School of Bioscience at the University of Skövde, as a final year Bachelor project toward the degree of Bachelor of Science in Cognitive Neuroscience. The project has been supervised by Judith Annett.

Reyes-Velázquez, W., \& Sealey-Potts, C., (2015). Unrealistic optimism, sex, and risk perception of type 2 diabetes onset : Implications for education programs. Feature Article, 28 (1), 1-9.

Rose, J., Endo, Y., Windschitl, P., \& Suls, J. ( 2008 ) . Cultural differences in unrealistic optimism and pessimism : The role of egocentrism and direct versus indirect comparison measures. The Society for Personality and Social Psychology, 34 ( 9 ), 1236-1248.

Seligman, M. (2006). Learned optimism: How to change Your mind and your life . London: Aazon.com.

Shepperd, A., Quellette, A., \& Fernandez, K. ( 1996). Abandoning unrealistic optimism : Performance estimates and the temporal proximity of self relevant feedback . Journal of Personality and Social Psychology, 70 (7),844-855

Shepperd, A., Waters, E., Weinstein, N., \& Klein, W. (2015). A primer on unrealistic optimism. Curr. Dir. Psychol. Sci., 24 (3), 232-237.

Suleiman, A. (2016). Differences in bullying and victimization between optimistic and non-optimistic adolescents, Studies in Educational Sciences, 43, 549-560. ( In Arabic)

Sutton, S. (2002). Influencing optimism in smokers by giving information about the average smoker. Risk Decision \& Policy, 7, 165-174.

Weinstein, D. (1980). Unrealistic optimism about future life events. Journal of Personality and Social Psychology, 39,806-820.

You, J., Fung, H., \& Isaacowitz, D.(2009) . Age differences in dispositional optimism: a cross-cultural study. Eur. J. Ageing, 6, 247-252.

Sereen, Z. (2011). The relationship of unrealistic optimism with healthy driving behavior for drivers. Unpublished Master Thesis, Faculty of Human, Social and Islamic Sciences, University of Haj Lakhdar, Algeria. ( In Arabic)

Sereen, Z. (2014). The relationship of unrealistic optimism with safe driving behavior, Amar Thulaiji University Studies, 30, 163-177. ( In Arabic)

First A. Author: Mokhtar Ahmad Al-Kayal

- Professor (Educational Psychology) Ain Shams University, Cairo, EGYPT, 26/9/2009.

- Former Head of Educational Psychology Department Faculty of Education, Ain Shams University (2013- 2019).

- Ph.D., (Educational Psychology) Ain Shams University, Cairo, EGYPT, Thesis Title "Physiological Brain Activity Associated With the Ability to Recall: A psycho- physiological Study".

- M.A., (Educational Psychology) Ain Shams University, Cairo, EGYPT, Thesis Title "The Relationship between Social Responsibility, Self-Concept and Locus of Control among College Student.

-Special Diploma (Education \& Psychology), Ain Shams University, Cairo, EGYPT 1983. 
- B.A.in Science \& Education (Biology) Ain Shams University, Cairo, EGYPT.

-Director of Psychological Counseling Center. Ain Shams University, Cairo, EGYPT (5/10/2010 - 5/ 10 /2012).

- Member of the Permanent Scientific Committee for the promotion of the degree of Professor and Assistant Professor specializing in Educational Psychology and mental health.

- Member of Arab association for Psychological Sciences.

- Member of Arab Association for Educational and Psychological Assessment.

- Member of Management council of Egyptian Association for Psychological studies.

- Member of the Egyptian Association for Psychological studies.

- Member of the Egyptian Psychological Association.

- Member of Association of Modern Education.

- Member of, Higher Committee for promotion (Level of professors, And Associate professors of Educational Psychology).

- Member of Higher Council of Culture, Ministry of Culture (Committee of Psychology).

- Member of Management Council of Faculty of Education, Ain shams University.

- Member of Management Council of Psychological Counseling Center, Faculty of Education, Ain shams University.

- Member of Undergraduate Students Affairs, Faculty of Education, Ain shams University.

- Member of Academic Counseling Committee, College of Humanities and Social Sciences, UAEU.

- Member of Postgraduate Students Affairs, Faculty of Education, Ain Shams University.

- Editor in chief of Psychological Counseling Journal, Faculty of Education, Ain shams University (Psychological Counseling Center).

- Editorial Board Member of many Local and Regional Journals of Psychology, as Examples, Journal of Psychological Studies, Issued by Faculty of Education, Ain Shams University,-Journal of Psychological Studies, issued by The Egyptian Association for Psychological Studies,-Arab Journal of Measurement, issued by Arab Association for Educational and Psychological Assessment, Journal of Psychological Measurement, Ain shams University (Psychological Measurement Center), and etc.

- Areas of Research Interest: Cognitive Psychology - Psychology of Learning - Physiological PsychologyEducational and Psychological Measurement.

\section{Second A.Author: Mohammad Ismail Sayed Hemeda}

- Place of birth: Al - Maqatfeya Village - Al Ayat Center - Giza Governorate, Date of Birth: 1/9/1975.

- Assistant Professor of Educational Psychology - Faculty of Education - Ain Shams University, 2015.

- Member of the Board of the Department of Educational Psychology, Faculty of Education, Ain Shams University.

- Member and Coordinator of the Quality Committee, Department of Educational Psychology - Faculty of Education, Ain Shams University.

- Member of the Environment and Society Committee, Faculty of Education, Ain Shams University.

- Member of the Egyptian Association for Psychological Studies.

- Bachelor of Arts and Education (Department of English) - (very good grade, the first), 1998.

- Professional diploma, specialization of school psychology (very good grade), 2001.

- Special Diploma in Education, Educational Psychology (very good grade), 2002.

- Master of Education (Educational Psychology) (excellence with the recommendation to exchange the message with the Egyptian and Arab universities and specialized research centers). Thesis Title: The impact of different characteristics of tasks and activities of the learner on the objectives of achievement goals among students in the preparatory stage, Faculty of Education - Ain Shams University, 2006.

- Ph.D. in Education (Educational Psychology) with a recommendation to exchange the message with the Egyptian and Arab universities and specialized research centers. Thesis title: The effectiveness of a program to develop reading comprehension in English for high school students, 2010, Faculty of Education - Ain Shams University.

Third A. Author: Mohammad Ali Haiba

- Born in Cairo, 1977.

- Member of the Egyptian Association for Psychological Studies, 2015.

- Professional Diploma, 2001.

- Special Diploma, 2002.

- Master in Educational Psychology, 2007.

- Ph.D. Educational Psychology, 2011.

- Assistant Professor of Educational Psychology, Faculty of Education, Ain Shams University. 
Fourth A. Author: Walid Hassan Ashour El Khateeb

- Born in Giza, 1981.

- Member of the Egyptian Association for Psychological Studies 2005- Professional Diploma, 2003.

- Special Diploma, 2005.

- Master in Educational Psychology, 2009.

- Ph.D. Educational Psychology, 2013.

- Lecturer of Educational Psychology, Faculty of Education, Ain Shams University, Jouf University. 